\title{
Controlled production of emulsion drops using an electric field in a flow-focusing microfluidic device
}

\section{Citation}

Kim, Haejune, Dawei Luo, Darren Link, David A. Weitz, Manuel Marquez, and Zhengdong Cheng. 2007. "Controlled Production of Emulsion Drops Using an Electric Field in a Flow-Focusing Microfluidic Device." Applied Physics Letters91 (13): 133106. https://doi.org/10.1063/1.2790785.

\section{Permanent link}

http://nrs.harvard.edu/urn-3:HUL.InstRepos:41511263

\section{Terms of Use}

This article was downloaded from Harvard University's DASH repository, and is made available under the terms and conditions applicable to Other Posted Material, as set forth at http:// nrs.harvard.edu/urn-3:HUL.InstRepos:dash.current.terms-of-use\#LAA

\section{Share Your Story}

The Harvard community has made this article openly available.

Please share how this access benefits you. Submit a story. 


\title{
Controlled production of emulsion drops using an electric field in a flow-focusing microfluidic device
}

\author{
Haejune Kim \\ Department of Mechanical Engineering, Texas A\&M University, College Station, Texas 77843, USA \\ Dawei Luo \\ Artie McFerrin Department of Chemical Engineering, Texas A\&M University, College Station, Texas 77843, \\ USA \\ Darren Link \\ Department of Physics and SEAS, Harvard University, Cambridge, Massachusetts 02138, USA and \\ RainDance Technologies, Guilford, Connecticut 06437, USA \\ David A. Weitz \\ Department of Physics and SEAS, Harvard University, Cambridge, Massachusetts 02138, USA \\ Manuel Marquez \\ Harrington Department of Bioengineering, Arizona State University, Tempe, Arizona 85287, USA and \\ Center for Integrated Nanotechnologies, Los Alamos National Laboratory, Los Alamos, New Mexico 87545, \\ USA \\ Zhengdong Cheng ${ }^{\text {a) }}$ \\ Artie McFerrin Department of Chemical Engineering, Texas A\&M University, College Station, Texas 77843, \\ USA
}

(Received 25 July 2007; accepted 8 September 2007; published online 26 September 2007)

\begin{abstract}
We describe a flexible emulsification method using an electric field to generate droplets in a hydrodynamic-flow-focusing geometry in microchannels. The droplet size is controlled by the ratio of inner and outer flow rates as well as by the electric field. As the voltage increases, the droplet size decreases. A Taylor cone is formed and generates very fine droplets, less than $1 \mu \mathrm{m}$ in diameter. Small inner flow rates and high electric fields are required to form a stable Taylor cone in a dc electric field. An ac electric field produces tiny droplets periodically. (C) 2007 American Institute of Physics. [DOI: 10.1063/1.2790785]
\end{abstract}

The technology of emulsion drop generation has a wide application in the food industry and in cosmetics, ${ }^{1}$ drug delivery, ${ }^{2}$ and ink-jet printing. Microfluidic technology provides a highly controllable means of emulsification. ${ }^{3-8}$ The flow focusing geometry ${ }^{3,8,9}$ is commonly used to generate droplets whose size is easily controlled by the flow rates. Anna and Mayer used a mode of breakup, the so-called tipstreaming method, to generate tiny droplets which are a few micrometers in diameter. ${ }^{10}$ However, there are limits to the smallest droplet size that can be achieved by changing the geometry, the size of the channels, and the properties of the fluids. One potential means of overcoming these limitations is the use of electrospray, an ionization technique that creates droplets in a high electric field; it has a wide range of applications such as mass spectrometry. ${ }^{11}$ Electrospraying is caused by an electric force applied to a liquid surface, and many experiments have been carried out to make smaller droplets using it. Bose reported electrohydrodynamic spraying from an electrified capillary in $1745 .^{12}$ In 1882, Rayleigh established a stability criterion for electrified droplets. ${ }^{13} \mathrm{Ze}$ leny carried out a pioneering systematic investigation of the discharge from the electrified water surface in $1917 .{ }^{14}$ Vonnegut and Neubauer computed the equilibrium value for the droplet radius by minimization of energy in 1952. ${ }^{15}$ In 1964 , Taylor described the cone-jet mode which has a semivertical angle of $49.3^{\circ} .{ }^{16}$ Mutoh studied the disintegration of a vis-

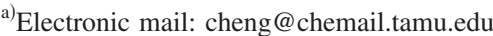

cous jet with various conductivities in $1979 .{ }^{17}$ In 1986 , Hayati et al. explained the mechanism of an electrospray using the electrical shear stress. ${ }^{18}$ However, only a few experiments of emulsification in an electric field have been performed with one liquid in another. In 1997, Sato et al. proposed a method to form droplets in water and explained electrohydrodynamic flow in macroscopic scale. ${ }^{19}$ However, the device was not able to produce an electrospray. Barrero et al. made cone jets of conducting liquids inside insulating liquids which are stationary. ${ }^{20}$

Here, we carried out electrospray in a microfluidic emulsification device using a flowing-focusing geometry. For this class of device, the droplet size depends on the size of the orifice, the geometry, the properties of the fluids, and flow rates of the inner and outer fluids; it is able to produce droplets as small as microns in size. ${ }^{9,10}$ Typically droplets are produced with a narrow size distribution $(\sim 2 \%)$. An electric field offers another means to control droplet size and possibly to generate submicron droplets.

We fabricated the device using soft lithography. ${ }^{21}$ The cross section of the channels is typically rectangular. The fluids are driven by syringe pumps. Distilled water is used as the dispersed phase which flows through the central channel. The conductivity of the water is $0.378 \mu \mathrm{S}$. The continuous phase is mineral oil with span of 80 (6 wt \%) added as a surfactant (Sorbitan mono-oleate, sigma, St. Louise, MO). The oil exerts a shear force on the water stream. Droplets are generated inside an orifice. The dispersed phase is always 

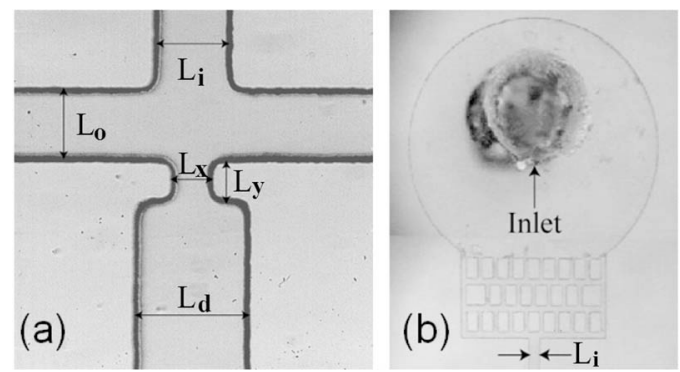

FIG. 1. Orifice and filter design. (a) Flow focusing geometry. $L_{i}=L_{o}$ $=100 \mu \mathrm{m}$ and $L_{d}=150 \mu \mathrm{m}$. The orifice size is $L_{x}=L_{y}=50 \mu \mathrm{m}$. (b) Micrograph of the filter, located at each inlet to prevent clogging.

surrounded by the continuous phase and thus is less likely to wet the walls of the channels than the continuous phase. Oil flow rates are normally higher than water flow rates to create a reasonable conic water tip. Drop sizes and the patterns of an assembly of droplets are controlled by the ratio of flow rates of inner to outer fluids. They are also affected by the geometry and the size of the channel. Smaller orifices can generate smaller droplets under identical operating conditions. The frequency of droplet production is on the order of $1 \mathrm{kHz}$; thus, it was captured using a high-speed chargecoupled device camera (PHANTOM v4.2, Vision Research, Wayne, NJ) with frame rates up to $2200 \mathrm{fps}$. We used a modified syringe pump (PHD2000, Harvard Apparatus, Holliston, MA) to generate stable flow at a very low rate, under $10 \mu \mathrm{l} / \mathrm{h}$, to overcome the fluctuations in the small flow rates found with the $1.8^{\circ}$ stepper motor. ${ }^{22}$ The stepping motion of the motor is much smoother using a reducer. A 100.56:1 speed reducer (Berg, East Rockaway, NY) was connected to the drive screw. While the motor turns 100.56 times, it generates a turn of the drive screw via the speed reducer. Thus, the drive screw connected to the syringe pump can develop a much smoother and uniform flow of the fluid.

After several experiments, the channels were easily clogged by the debris from the holes punched in the polydimethylsiloxane (PDMS) using modified stunt syringe needles. To overcome this, we designed filters [Fig. 1(b)] in each inlet; they had a smaller width than the channels, preventing debris from entering. Channels were coated by Aquapel (PPG Industries, Inc., Pittsburgh, PA) to prevent water droplets from wetting the walls. The coating makes the surfaces more hydrophobic. The height of the channels was $61.4 \mu \mathrm{m}$ as measured by a profilometer. The liquid should be slightly conducting to form a Taylor cone. ${ }^{18}$ If the liquid has a high conductivity, it cannot form a Taylor cone because there would be no potential difference in the liquid.

The source and ground electrodes were inserted into the PDMS and they contacted the fluid inside the channels; this generated an electric field between the electrodes. The electric field depends on the distance $(0.3645 \mathrm{~mm})$ between the electrodes as well as the applied potential difference.

The formation of a Taylor cone is necessary to generate very fine droplets. When an electric field is applied, the water-oil interface at the tip is charged and behaves as a capacitor. As the voltage increases, the charges on the interface increase resulting in a higher attraction downstream. The tip of the Taylor cone is stretched to a narrow filament and is broken into tiny droplets due to the Rayleigh instability. ${ }^{23}$ the eyes.
Downloaded 30 Sep 2007 to 128.103.60.225. Redistribution subject to AlP license or copyright, see http://apl.aip.org/apl/copyright.jsp (a) $5 / 350$

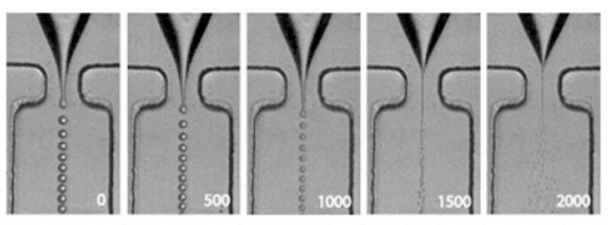

(b) $20 / 350$
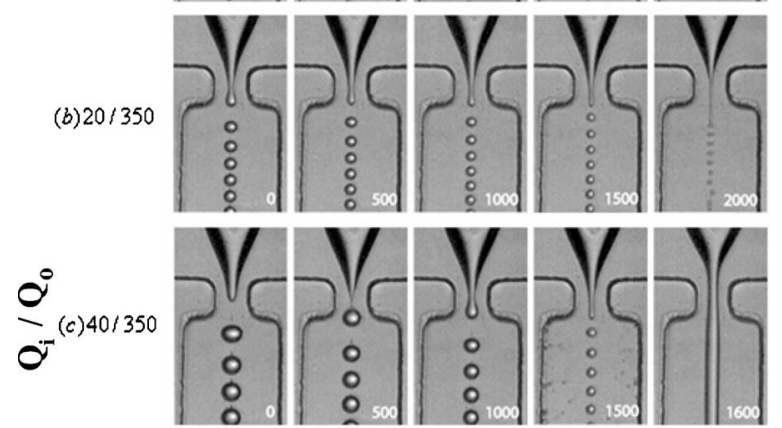

(d) $60 / 350$

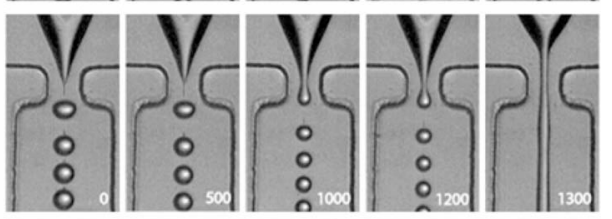

(e) $80 / 350$

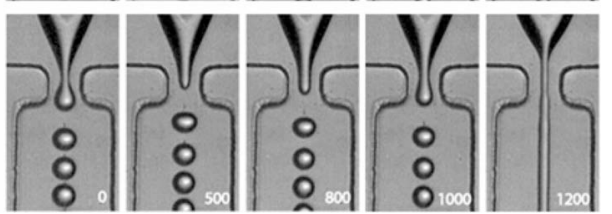

FIG. 2. Phase diagram of droplet generation in a dc electric field. Each row represents different flow-rate ratios of inner and outer fluids $\left(Q_{i} / Q_{o}\right)$. The number at the corner of each micrograph represents the applied voltage.

Smaller droplet sizes are produced at smaller ratios of flow rates of dispersed fluid to continuous fluid, at the same voltage. The smallest droplets, under $1 \mu \mathrm{m}$ in diameter, were produced when the tip formed a Taylor cone, as shown in Fig. 2(a). Low flow-rate ratios, as in case (a), are necessary to form a Taylor cone. The electric field barely affects the droplet size at high flow-rate ratios such as in case (e). A jet is formed at $1200 \mathrm{~V}$ and did not generate droplets. Jetting appears at high voltage and high flow-rate ratios. The tip of water bridged the ground electrode and no longer generated droplets. Because of the jetting, we were unable to achieve a Taylor cone at any voltage.

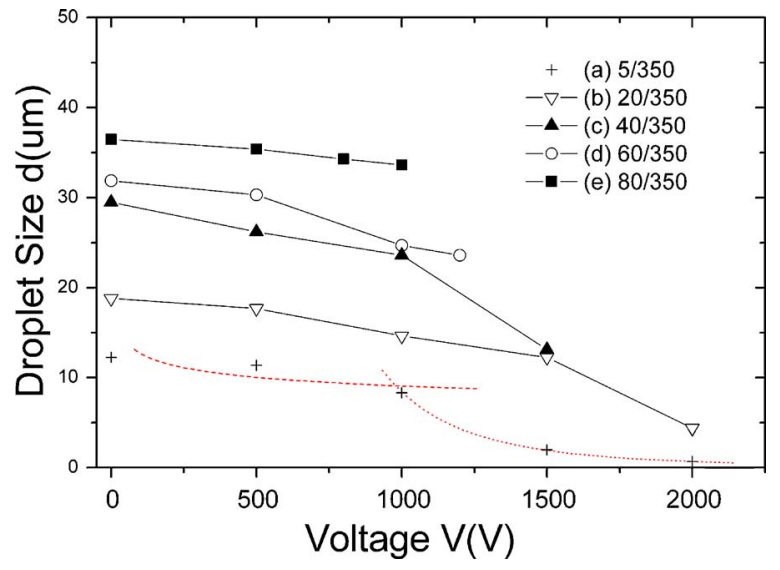

FIG. 3. (Color online) Drop-size dependence on voltage and flow-rate ratio. The data for case (a) are fit to power functions. The solid lines are guides to 

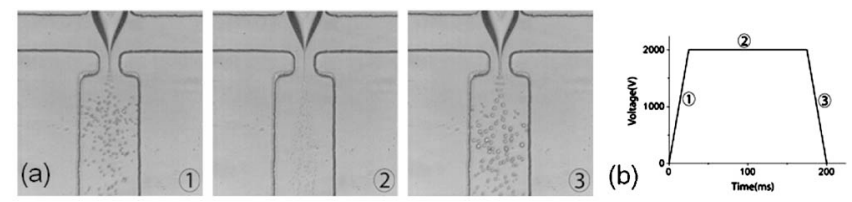

FIG. 4. Periodic formation of a Taylor cone under an ac electric field. (a) Micrograph showing drop formation at the ramp and peak of a rectangular waveform. (b) Rectangular waveform. Flow-rate ratio $\left(Q_{i} / Q_{o}\right), 5 / 350$ and voltage, $2000 \mathrm{~V}$.

Quantitative information from Fig. 2 is extracted by image analysis and plotted in Fig. 3. At a large flow-rate ratio, the droplet sizes are of the same order as the orifice. Decreasing the flow-rate ratio, the droplet sizes can decrease by half an order of magnitude due to the flow focusing. Case (a) shows that a decrease of an additional order of magnitude can be achieved using an electric field. Electrospray with a Taylor cone was observed for case (a) above $1500 \mathrm{~V}$. Drop size was related to applied voltages as $d \sim V^{-0.2 \pm 0.1}$ at low voltage when flow focusing dominates and as $d \sim V^{-3.6 \pm 0.1}$ at high voltages when the electric field dominates. The crossover voltage for case (a) is $1000 \mathrm{~V}$ (the corresponding field strength is about $2 \mathrm{~V} / \mu \mathrm{m}$ ). The observed dependence of size on voltage is consistent with theory. ${ }^{24}$ While the electric field is applied, the droplets are deformed, becoming stretched in the direction of the electric field; enhanced coalescence was then observed.

At low water flow rate, the Taylor cone remained only for about a minute with a dc field because of variations in the flow rate from the syringe pump. To obtain a more stable emulsification mode at high voltage, we used an ac field. A rectangular waveform was using a function generator (DS345, Stanford Research Systems, Sunnyvale, CA). Dropsize distributions at the ramps [Fig. 4(a), (1), and (3)] were wide. When the applied voltage reached the peak [Fig. 4(b), (2)], it generated tiny droplets until the voltage-decreasing ramp was reached. The drops from the Taylor cone at $2000 \mathrm{~V}$ are periodic with the waveform. A means to separate the drops would be required to collect them.

In conclusion, we have demonstrated the integration of an electric field with a flow-focusing geometry using a microfluidic device. The size of the emulsion droplet produced was controlled by the electric field in microchannels. At low voltage and low flow-rate ratio, the size is determined solely by hydrodynamic-flow focusing. The electric field can pre- cisely control the droplet size only at small flow-rate ratios with a low flow rate of the dispersed phase. The droplet size produced by the Taylor cone is less than $1 \mu \mathrm{m}$. In the electrospray regime where the electric field is larger than the formation threshold for a Taylor cone, the flow rate of the dispersed phase must be very small to avoid coalescence of the droplets in the channel. We also used an ac field to demonstrate the periodic formation of a Taylor cone.

We thank the support from PM USA INEST program and the start-up fund from Texas Engineering Experimental Station and Texas A\&M University. D.A.W. is supported by the NSF No. (DMR-0602684).

${ }^{1}$ M. Chappat, Colloids Surf., A 91, 57 (1994).

${ }^{2}$ P. S. Dittrich and A. Manz, Nat. Rev. Drug Discovery 5, 210 (2006).

${ }^{3}$ A. M. Ganan-Calvo and J. M. Gordillo, Phys. Rev. Lett. 87, 274501 (2001).

${ }^{4}$ G. R. Yi, T. Thorsen, V. N. Manoharan, M. J. Hwang, S. J. Jeon, D. J. Pine, S. R. Quake, and S. M. Yang, Adv. Mater. (Weinheim, Ger.) 15, 1300 (2003).

${ }^{5}$ D. R. Link, S. L. Anna, D. A. Weitz, and H. A. Stone, Phys. Rev. Lett. 92, 054503 (2004).

${ }^{6}$ D. R. Link, E. Grasland-Mongrain, A. Duri, F. Sarrazin, Z. D. Cheng, G. Cristobal, M. Marquez, and D. A. Weitz, Angew. Chem., Int. Ed. 45, 2556 (2006).

${ }^{7}$ H. Song, D. L. Chen, and R. F. Ismagilov, Angew. Chem., Int. Ed. 45, 7336 (2006).

${ }^{8}$ T. Y. Gong, J. Y. Shen, Z. B. Hu, M. Marquez, and Z. D. Cheng, Langmuir 23, 2919 (2007).

${ }^{9}$ S. L. Anna, N. Bontoux, and H. A. Stone, Appl. Phys. Lett. 82, 364 (2003).

${ }^{10}$ S. L. Anna and H. C. Mayer, Phys. Fluids 18, 121512 (2006).

${ }^{11}$ J. B. Fenn, M. Mann, C. K. Meng, S. F. Wong, and C. M. Whitehouse, Science 246, 64 (1989).

${ }^{12}$ A. G. Bailey, Electrostatic Spraying of Liquids (Wiley, New York, 1988).

${ }^{13}$ L. J. W. S. Rayleigh, Philos. Mag. 44, 184 (1882).

${ }^{14}$ J. Zeleny, Phys. Rev. 3, 69 (1914).

${ }^{15}$ B. Vonnegut and R. L. Neubauer, J. Colloid Sci. 7, 616 (1952).

${ }^{16}$ G. Taylor, Proc. R. Soc. London, Ser. A 280, 383 (1964).

${ }^{17}$ M. Mutoh, S. Kaieda, and K. Kamimura, J. Appl. Phys. 50, 3174 (1979).

${ }^{18}$ I. Hayati, A. I. Bailey, and T. F. Tadros, Nature (London) 319, 41 (1986).

${ }^{19}$ M. Sato, T. Hatori, and M. Saito, IEEE Trans. Ind. Appl. 33, 1527 (1997).

${ }^{20}$ A. Barrero, J. M. Lopez-Herrera, A. Boucard, I. G. Loscertales, and M. Marquez, J. Colloid Interface Sci. 272, 104 (2004).

${ }^{21}$ J. C. McDonald, D. C. Duffy, J. R. Anderson, D. T. Chiu, H. K. Wu, O. J. A. Schueller, and G. M. Whitesides, Electrophoresis 21, 27 (2000).

${ }^{22}$ M. A. Holden, S. Kumar, E. T. Castellana, A. Beskok, and P. S. Cremer, Sens. Actuators B 92, 199 (2003).

${ }^{23}$ L. J. W. S. Rayleigh, Proc. London Math. Soc. 14, 170 (1883).

${ }^{24}$ A. M. Ganan-Calvo, Phys. Rev. Lett. 98, 134503 (2007). 